УДК

\title{
КОНСТРУКЦИЯ ГЕНЕРАТОРА Х-ДИАПАЗОНА С ДИЭЛЕКТРИЧЕСКИМ РЕЗОНАТОРОМ И ОТРИЦАТЕЛЬНОЙ ПРОВОДИМОСТЬЮ*
}

\author{
С. С. ОЛОКИД, С. Б. ЗАКИ, Н. М. МАХЮДДИН, М. Ф. АИН, З. А. АХМАД
}

Малазийский университет наук, Малайзия, Нибонг Тебал, Пинанг

\begin{abstract}
Аннотация. В статье разработан и реализован планарный перестраиваемый СВЧ-генератор Х-диапазона с низким уровнем фазового шума, использующий диэлектрический резонатор (ДР) с оригинальным питанием и одним транзистором. Предлагается керамический ДР на основе сложного оксида ZrSnTi с диэлектрической проницаемостью, равной 95, который заключен в металлическую полость и имеет собственную добротность, равную 5000 на частоте 10 ГГц. Связь резонансной частоты с геометрическими параметрами устанавливается путем использования метода компенсации на основе двойной обратной связи с отрицательной проводимостью, выходы которой объединяются посредством делителя мощности Вилкинсона. Генератор с ДР и обратной связью с параллельными линиями связи встраивается в многослойную СВЧ-плату посредством использования технологии фотолитографии. Этот генератор включает псевдоморфный малошумящий усилитель на базе рНЕМТ транзистора. Проведены измерения предлагаемого генератора с механической подстройкой. Полученные результаты показывают, что ДР резонирует на волне типа $T E_{01 \delta}$ с частотой 10 ГГц. Измеренный фазовый шум генератора составил $-81,03$ дБн/Гц при смещении 100 кГц.
\end{abstract}

Ключевые слова: генератор с диэлектрическим резонатором; обратная связь; отрицательная проводимость; параллельная связь; фазовый шум

\section{1. ВВЕДЕНИЕ}

Стремление к улучшению рабочих характеристик и эффективных альтернативных решений заставило специалистов по разработке систем искать новые оригинальные продукты, которые способны обеспечить более высокие рабочие характеристики системы при невысокой стоимости. Другие цели разработки включают снижение потребляемой мощности и уменьшение размеров на $30 \%$ [1].
Современная потребность в реализации эффективной миниатюрной интегральной схемы СВЧ диапазона MMIC (miniaturized microwave integrated circuit) обусловливает особое внимание к достижению низкого уровня шума, малых размеров, дешевизны, высокой эффективности, высокой температурной устойчивости и высокой надежности. Дополнительной важной проблемой для системотехников, особенно при выборе конструкции генератора, является фазовый шум.

* $\quad$ Авторы выражают признательность университету Sains Malaysia за финансовую поддержку в рамках университетского исследовательского гранта № 1001/PELECT/854004. 


\section{БИБЛИОГРАФИЧЕСКИЙ СПИСОК}

1. Razavi, B. Challenges in portable RF transceiver design. IEEE Circuits Devices Mag., vol. 12, no. 5, pp. 12-25, Sept. 1996. DOI: 10.1109/101.537352.

2. GED 2013. Dielectric Resonator Oscillator (DRO Information). General Electronic Devices. URI: http:// www.gedlm.com/DRO.

3. Wan, J. Design of a $5.305 \mathrm{GHz}$ dielectric resonator oscillator with simulation and optimization. J. Electronic Science Technol. China, vol. 6, No. 3, pp. 342-345, 2008. URI: http://manu50.magtech.com.cn/Jwk jest/EN/Y200 8/V6/I3/342.

4. Yan, Guoguang. The design of the $\mathrm{Ku}$ band dielectric resonator oscillator. Proc. of Int. Conf. on Electronic Packaging Technology \& High Density Packaging, ICEPT-HDP, 28-31 Jul. 2008, Shanghai, China. IEEE, 2008, pp. 1-3. DOI: 10.1109/ICEPT.2008. 4607000.

5. Sun, B.; Wu, Y.; Luo, B.; Du, G. Design of 5.8 $\mathrm{GHz}$ dielectric resonator oscillator applied in electronic toll collection. Proc. of 5 Int. Conf. on Wireless Communications, Networking and Mobile Computing, WiCom, 24-26 Sept. 2009, Beijing, China. IEEE, 2009, pp. 1-3. DOI: 10.1109/WICOM.2009.5303409. 
6. Tanaka, T.; Aikawa, M. A K-band push-push oscillator using dielectric resonator. Proc. of 13 Int. Symp. on Antenna Technology and Applied Electromagnetics and the Canadian Radio Science Meeting, ANTEM/URSI, 15-18 Feb. 2009, Toronto, ON, Canada. IEEE, 2009, pp. 1-4. DOI: 10.1109/ANTEMURSI.2009. $\underline{4805120 .}$.

7. Y. Du, Z.-X. Tang, B. Zhang, P. Su, "K-band harmonic dielectric resonator oscillator using parallel feedback structure," PIER Lett., Vol. 34, p. 83-90, 2012. DOI: 10.2528/PIERL12061108.

8. Liu, Han-Li; Wang, Lang; Lin, Guo-Wei. Design of a K-band push-push dielectric resonator oscillator. Proc. of Int. Workshop on Electromagnetics; Applications and Student Innovation, iWEM, 6-9 Aug. 2012, Chengdu, Sichuan, China. IEEE, 2012, pp. 1-2. DOI: 10.1109/ iWEM.2012.6320369.

9. Xia, Q.; Tang, Z.; Zhang, B. Design of a $17.4 \mathrm{GHz}$ push-push dielectric resonator oscillator. Proc. of Int. Conf. on Microwave and Millimeter Wave Technology, ICMMT, 8-11 May 2010, Chengdu, China. IEEE, 2010, pp. 532-535. DOI: 10.1109/ICMMT.2010.5525221.

10. Su, P.; Tang, Z.-X.; Zhang, B. Push-push dielectric resonator oscillator using substrate integrated waveguide power combiner. PIER Lett., Vol. 30, p. 105-113, 2012. DOI: 10.2528/PIERL11122302.

11. Fusco, V. F.; Dearn, A. Dielectric Resonator Oscillators. Wiley Encyclopedia of Electrical and Electronics Engineering, 1999

12. Sinnesbichler, F. X.; Hautz, B.; Olbrich, G. R. A $\mathrm{Si} / \mathrm{SiGe} \mathrm{HBT}$ dielectric resonator push-push oscillator at $58 \mathrm{GHz}$. IEEE Microwave Guided Wave Lett., vol. 10, no. 4, pp. 145-147, Apr. 2000. DOI: 10.1109/75.846927.

13. Ishihara, O.; Mori, T.; Sawano, H.; Nakatani, M. A highly stabilized GaAs FET oscillator using a dielectric resonator feedback circuit in 9-14 GHz. IEEE Trans. Microwave Theory Tech., vol. 28, no. 8, pp. 817-824, Aug 1980. DOI: 10.1109/TMTT.1980.1130177.

14. Abe, H.; Takayama, Y.; Higashisaka, A.; Takamizawa, H. A highly stabilized low-noise GaAs FET integrated oscillator with a dielectric resonator in the $\mathrm{C}$ band. IEEE Trans. Microwave Theory Tech., vol. 26, no. 3, pp. 156-162, Mar 1978. DOI: 10.1109/TMTT.1978. $\underline{1129336}$.

15. Ogawa, K.; Ikeda, H.; Ishizaki, T.; Hashimoto, K.; Ota, Y. $25 \mathrm{GHz}$ dielectric resonator oscillator using an AlGaAs/GaAs HBT. Electron. Lett., vol. 26, no. 18, pp. 1514-1516, 30 Aug. 1990. DOI: 10.1049/el:19900972.
16. Llopis, O.; Dienot, J.-M.; Verdier, J.; Plana, R.; Gayral, M.; Graffeuil, J. Analytic investigation of frequency sensitivity in microwave oscillators: Application to the computation of phase noise in a dielectric resonator oscillator. Annales Des Télécommunications, Vol. 51, No. 3-4, pp. 121-129, 1996. DOI: $10.1007 / \mathrm{BF} 02995502$.

17. Herczfeld, P. R.; Daryoosh, A.; D’Ascenzo, C.; Contarino, M.; Rosen, A. Optically tuned and FM modulated X-band dielectric resonator oscillator. Proc. of 14th European Microwave Conf., 10-13 Sept. 1984, Liege, Belgium. IEEE, 1984, pp. 268-273. DOI: 10.1109/EUMA. 1984.333407.

18. Makino, T.; Hashima, A. A highly stabilized MIC Gunn oscillator using a dielectric resonator. IEEE Trans. Microwave Theory Tech., vol. 27, no. 7, pp. 633-638, Jul. 1979. DOI: 10.1109/TMTT.1979.1129692.

19. Mizan, M. A.; Sturzebecher, D.; Higgins, T.; Paolella, A. An X-band, high power dielectric resonator oscillator for future military systems. IEEE Trans. Ultrasonics, Ferroelectrics, Frequency Control, vol. 40, no. 5, pp. 483-487, Sept. 1993. DOI: 10.1109/58.238099.

20. Kishk, A. A.; Glisson, A. W.; Junker, G. P. Bandwidth enhancement for split cylindrical dielectric resonator antennas. PIER, Vol. 33, pp. 97-118, 2001. DOI: 10.2528/PIER00122803.

21. Petosa, A. Dielectric Resonator Antenna Handbook. Artech House, 2007.

22. Shen, T.; Zaki, K. A.; Wang, C. Tunable dielectric resonators with dielectric tuning disks. IEEE Trans. Microwave Theory Tech., vol. 48, no. 12, pp. 2439-2445, Dec. 2000. DOI: 10.1109/22.898995.

23. Shen, T.; Zaki, K. A.; Wang, C.; Deriso, J. Tunable dielectric resonators with dielectric tuning disks in cylindrical enclosures. Proc. of IEEE MTT-S Int. Microwave Symp. Dig., 11-16 June 2000, Boston, MA, USA. IEEE, 2000, vol. 3, pp. 1441-1444. DOI: $\underline{10.1109 /}$ MWSYM.2000.862245.

24. Vendelin, G. D.; Pavio, A. M.; Rohde, U. L. Microwave Circuit Design Using Linear and Nonlinear Techniques. New York: Wiley, 1990.

25. Vendelin, G. D.; Pavio, A. M.; Rohde, U. L. Microwave Circuit Design Using Linear and Nonlinear Techniques, 2nd ed. Hoboken, New Jersey: John Wiley \& Sons, Inc., 2005. DOI: 10.1002/0471715832.

26 Bahl, I. J.; Bhartia, P. Microwave Solid State Circuit Design. New York: Wiley, 1988. 